\title{
Un viaje a través de mí misma. Autoexpresión y plenitud vital en la danza
}

\author{
LORELCRIS RENGIFO RODRIGUEZ \\ al282846@uji.es \\ ViRginia CARRERo Planes \\ carrero@uji.es
}

\section{Resumen}

Introducción: Diversos estudios muestran la influencia del trabajo corporal sobre variables psicológicas tales como la inteligencia emocional, las competencias sociales y el pensamiento creativo, lo cual derivó el interés por conocer los efectos de la danza sobre las habilidades emocionales en relación con la plenitud y el bienestar. La presente investigación tiene por objetivo explorar la vivencia de personas que practican danza en relación con su percepción de bienestar psicoemocional y plenitud vital. Método: Los datos se han recolectado mediante entrevistas en profundidad a nueve (9) personas dedicadas a la danza o actividades de movimiento y mediante revisión de fuentes secundarias de información. El análisis de datos se ha hecho siguiendo la metodología grounded theory (Glaser y Strauss, 1967) enmarcada en aproximaciones cualitativas constructoras de teoría, aplicando las técnica de muestreo teórico, el método comparativo constante y la saturación teórica de categorías. Resultados: Se han obtenido un conjunto de categorías relacionadas con el bienestar psicoemocional y la plenitud vital (conciencia de sí mismo, conexión con otras personas, actualización del potencial personal, despertar de la vitalidad, expresión de emociones y comunicación), que son desarrolladas mediante ciertos procesos intrínsecos a la práctica de la danza (la atención plena, el insight corporal y la libertad en el movimiento). Conclusiones: Los elementos identificados presentes en la práctica de la danza actúan como facilitadores para la autoexpresión y la plenitud vital, desde la vivencia de una experiencia integradora entre cuerpo y mente, la ampliación de la percepción de las propias capacidades y el aumento de la valía personal.

Palabras clave: danza, plenitud, sentido vital, bienestar psicoemocional, autoexpresión, grounded theory.

\footnotetext{
Abstract

Introduction: Many studies have identified the influence of corporal work on different psychological variables such as emotional intelligence, social competences and creative thinking, this led the interest for knowing the effects or benefits of dancing on the emotional abilities in relation with plenitude and wellness. This investigation has the objective to explore the experience of people that practice dance in relation with their perception
} 
of psychoemotional wellness and vital plenitude. Method: The recollection of data has being realized through deep interviews to nine persons dedicated to dancing activities or related with movement, and a review of secondary sources of information. The analyses of the data has being done by grounded theory (Glaser y Strauss, 1967) framed within qualitative approximations constructors of theory, applying the theoretical sampling, the systematic use of the constant comparative method and the theoretical saturation of categories. Results: The results obtained have shown a group of related categories with the psychoemotional wellness and vital plenitude (awareness of one self, connection with others, update of personal potential, awakening of the vitality, emotional expression and communication), which are developed through out certain intrinsic processes of dance practices (full attention, corporal insight and liberty in the movement). Conclusions: These elements facilitate the self-expression and vital plenitude, such as the living of an integrative experience of body and mind, the widening of the perception of the own capacities and the enhancing of the self-value.

Keywords: dance, plenitude, vital sense, psychoemotional wellness, self-expression, grounded theory.

\section{Introducción}

Las últimas investigaciones en neurociencia y los avances de un conjunto de disciplinas llamadas ciencias del desarrollo somático (somatic movement sciences) han apoyado, especialmente desde los años 70 , la idea de que un apropiado desarrollo motor y corporal tiene impacto en las funciones de diferentes partes del cerebro (Rizzolatti y Sinigaglia, 2006). La condición humana integra dos aspectos que están íntimamente relacionados, cuerpo y mente. Debido a esta unión, los cambios en el repertorio de movimientos pueden producir cambios en el nivel de la mente (Payne, 1990; Ratey, 2001). Estas ideas están apoyadas en teorías como la del embodiment, la cual señala la existencia de una modalidad perceptiva especifica de representación del conocimiento, una modalidad guiada desde el sistema sensorio-motor y el uso de este sistema para pensar a través de las experiencias encarnadas (Koch, 2006).

El procesamiento, la representación y la percepción de señales corporales (interocepcion y propiocepcion) juegan un importante rol en la conducta humana. Las teorías de la cognición encarnada (embodied cognition) sostienen que la mayoría de los procesos cognitivos operan mediante símbolos perceptuales y el uso de este concepto implica la reactivación de los estados sensorio-motores que ocurren durante las experiencias con el mundo. Similarmente, la activación de las representaciones interoceptivas y las meta-representaciones de las señales corporales apoyan la toma de conciencia interoceptiva que está profundamente asociada con las experiencias emocionales y el funcionamiento cognitivo (Herbert y Pollatos, 2012).

Las ideas expuestas desde el embodiment apuntan a la ideoneidad de la danza para la toma de consciencia de las señales sensorio-motoras, ya que su práctica se basa en el movimiento, el cual genera una serie de sensaciones interocepctivas y propioceptivas (SheetsJohnstone, 2010)

Por otra parte, se han realizado algunos estudios que resaltan los beneficios del movimiento, el ejercicio físico y la danza. Dentro del ámbito educativo se ha encontrado que la implementación de programas de baile y danza creativa mejora las competencias sociales y la conducta (Lobo y Winsler, 2006) y el pensamiento creativo (Jay, 1991) y facilita el desarro- 
llo de los niños (Mac Donald, 1991). En adolescentes y jóvenes que practican danza se ha observado que la misma actúa como un medio de expresión, de comunicación, de promoción comunitaria, de utilización del tiempo libre, como ejercicio espiritual, como medio de socialización y como agente educativo (Peña y Jaramillo, 1998).

También se ha observado cierta relación con la inteligencia emocional, como en el caso de la investigación de López y De Rueda (2013) en el que estudiantes universitarios mostraron mejoras significativas de los factores de percepción y compresión emocional tras la aplicación de un programa basado en recursos dancísticos y patrones musicales. Igualmente se han evaluado las repercusiones del ejercicio físico y el movimiento regular, observándose que actúan como factor protector ante el deterioro de habilidades emocionales y favorecedor de estados emocionales positivos y estilos de respuesta más adaptativos ante trastornos como la depresión (Lima de Figueiredo Queirós, Fernández Berrocal, Extremera y Susana Queirós, 2006).

Al examinar los efectos de disciplinas que emplean la danza desde un enfoque terapéutico, se ha visto que la aplicación de un programa motor basado en la biodanza generó un aumento significativo de los niveles de inteligencia emocional percibido en mujeres (Abad, Castillo y Orizia, 2014). El desarrollo de un programa de psicoterapia del movimiento influyó de manera positiva en la comunicación de emociones de pacientes adolescentes esquizofrénicos (Salas-Calderón, 2007).

La danza movimiento terapia ha demostrado promover el incremento de aptitudes físicas y motoras, la exteriorización de emociones y una mejor y mayor capacidad de integración social al potenciar la cooperación en grupos (Martínez, J., Martínez, L., García y MartínezAlmagro, 2004). El uso de esta técnica en casos de enfermedades crónicas ha ayudado a aliviar efectos colaterales de las terapias tradicionales, reduciendo la fatiga, la ansiedad, la depresión y la percepción del dolor y aumentando el vigor, la energía vital y mejorando la autoimagen y los estados internos positivos del paciente (Sebiani, 2005). Su práctica en entornos laborales ha influido en la mejora de la empatía, de las habilidades relacionales, de la inteligencia emocional, del bienestar psicológico y de la satisfacción vital (Fischman, 2005, c. p. Rodríguez, Caja y Gracia, 2013).

Tal como se puede ver a partir de las investigaciones reseñadas, la danza y el movimiento tienen una influencia positiva en la salud física y psicológica del individuo, siendo además un modo de expresión que ha formado parte de la cultura desde el principio de la vida en sociedad y que se mantiene aún en nuestros tiempos, demostrando su importancia en la vida humana. Desde esta perspectiva, y teniendo en cuenta las evidencias referidas sobre los efectos positivos de la danza, la presente investigación se focaliza en comprender los mecanismos mediante los que la práctica de danza favorece y desarrolla el bienestar psicoemocional y la vivencia de plenitud.

Partiendo de esta inquietud, el trabajo que se presenta tiene como objetivo general explorar la vivencia de personas que practican danza en relación a su percepción de plenitud y bienestar psicoemocional. Para alcanzar dicho objetivo se contempla, por una parte, reconocer el impacto que la práctica de la danza tiene sobre la plenitud y el bienestar psicoemocional $y$, en segundo lugar, identificar los mecanismos (procesos psicosociales) por los que la práctica de la danza influye en el desarrollo de la plenitud y el bienestar psicoemocional.

Finalmente, se pretende aportar información para generar directrices en la aplicación de acciones terapéuticas basadas en el movimiento para el desarrollo de la autoexpresión y la plenitud vital. 


\section{Método}

\section{Metodología de análisis}

Se empleó la grounded theory o teoría fundamentada (Glaser y Strauss, 1967) enmarcada en aproximaciones cualitativas constructoras de teoría. La teoría fundamentada «es una metodología de análisis, unida a la recogida de datos, que utiliza un conjunto de métodos, sistemáticamente aplicados, para generar una teoría inductiva sobre un área sustantiva» (Glaser, 1992: 16, c. p. Carrero, Soriano y Trinidad, 2012).

El análisis se realiza empleando las siguientes técnicas (Carrero, Soriano y Trinidad, 2012):

- El muestreo teórico: empleado para coleccionar, codificar y analizar los datos y decidir qué nuevos datos integrar y dónde encontrarlos para desarrollar una teoría mejor a medida que se va perfeccionando. No se comparan poblaciones, sino características de creación de ideas, que a su vez delimitan patrones comportamentales y actitudinales.

- El método comparativo constante (en adelante, MCc) consiste en la comparación para encontrar semejanzas y diferencias a través del análisis de los incidentes contenidos en los datos, para generar conceptos y sus características, basados en patrones del comportamiento que se repiten.

- La saturación teórica (en adelante, ST) es el criterio por el cual el investigador decide cesar el muestreo de los distintos grupos, pertinentes a cada una de las categorías. Significa que no se ha encontrado ninguna información adicional que permita desarrollar nuevas propiedades de la categoría o una nueva categoría.

\section{Técnica de recolección de datos}

Se ha empleado la técnica de entrevista en profundidad, que busca obtener descripciones del mundo de la vida del entrevistado con respecto a la interpretación del significado de los fenómenos descritos (Kvale, 2011).

\section{Muestreo}

Se aplicó un muestreo no probabilístico que siguió los criterios del muestreo teórico, para garantizar la representatividad de las categorías generales surgidas. Fueron seleccionadas 9 personas ( 7 mujeres, 2 hombres) con edades comprendidas entre 26 y 41 años, que realizan actividades relacionadas con la danza y el movimiento bien de manera profesional o por afición (ballet, danza contemporánea, mimo corporal dramático, pilates, yoga, biodanza, danza movimiento terapia, movimiento autentico o danza del vientre).

\section{Procedimiento}

Se realizaron 9 entrevistas en profundidad. Las primeras 3 entrevistas se plantearon con el fin de realizar un estudio exploratorio y en función del marco teórico producto de la revisión bibliográfica. Lo datos fueron analizados aplicando el MCC, emergiendo categorías y propieda- 
des que sirvieron de base para guiar las entrevistas posteriores, resaltando el papel del movimiento dentro de la danza. Así mismo, se revisó material bibliográfico que fue tomado como dato secundario, analizado y categorizado, destacando el rol del movimiento como aspecto facilitador de la experiencia.

Las siguientes 3 entrevistas en profundidad se realizaron a personas no solo relacionadas con la danza sino también con otras actividades de movimiento, esto con la intención de ampliar la variabilidad de los datos y ensanchar la interpretación de los aspectos sobresalientes en los análisis anteriores. Los datos recabados fueron analizados permitiendo nutrir las categorías ya definidas y vislumbrar algunas nuevas, apuntando a la importancia del movimiento consciente como elemento facilitador para la percepción de bienestar.

Posteriormente se entrevistaron 2 personas con discapacidad que practican danza, con la finalidad de indagar más sobre el aspecto del movimiento como facilitador de la experiencia y el proceso de expansión del yo en este tipo de población. Así mismo, se entrevistó a una persona formada en danza contemporánea con experiencia en movimiento autentico (técnica centrada en la búsqueda de patrones de movimientos espontáneos) para identificar los matices que aportan cada uno de estos tipos de práctica de danza y movimiento.

En la figura 1 se muestra el diseño resultante de la aplicación de la teoría fundamentada en el presente estudio.

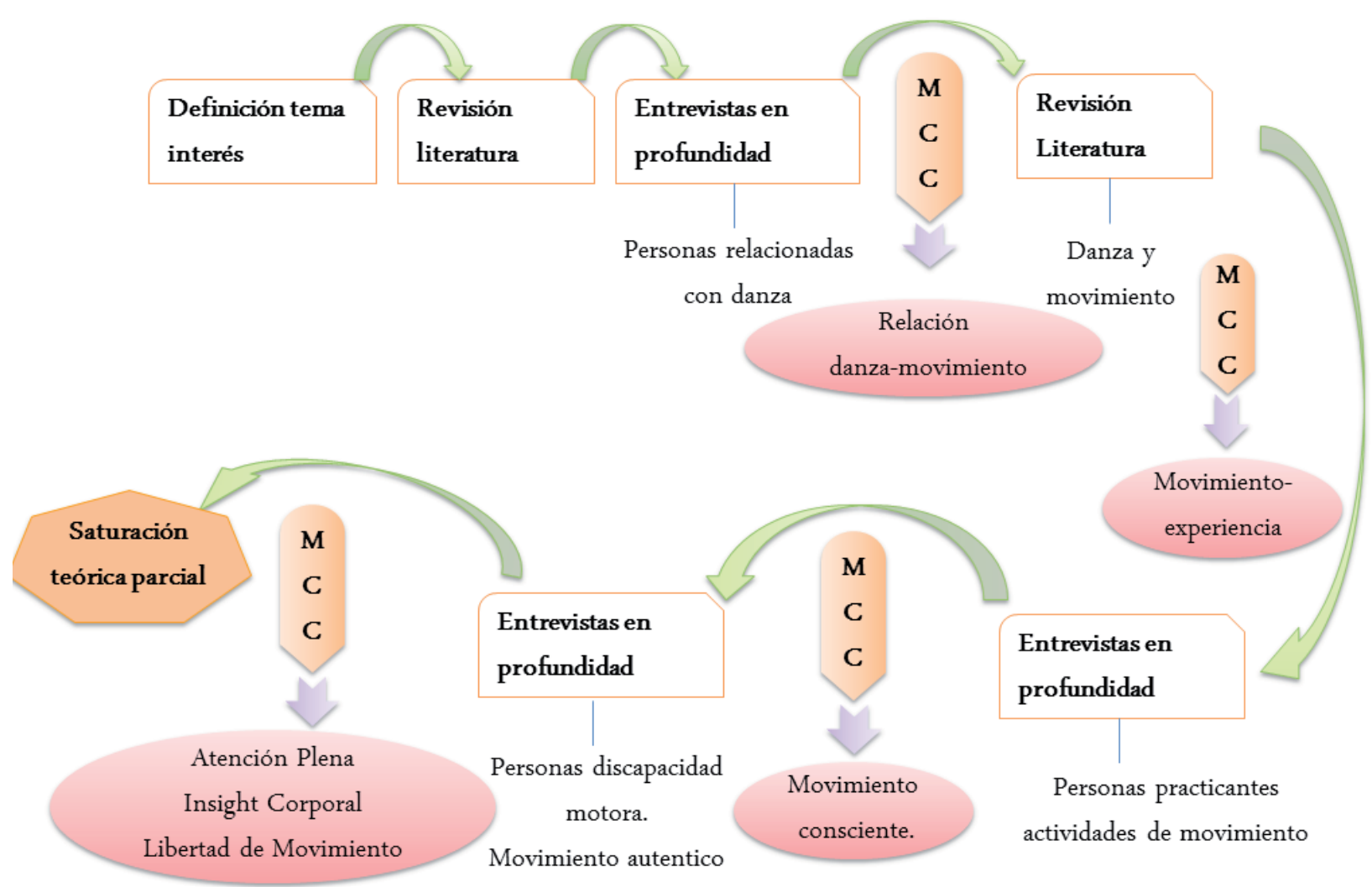

Figura 1. Aplicación de la metodología grounded theory 


\section{Resultados}

A continuación se presentan los resultados obtenidos producto de la aplicación del MCC y de la ST parcial de las categorías, emergidas a raíz del análisis de la información contenida en las entrevistas realizadas. Se exponen las categorías con las propiedades representativas de cada una y las relaciones que se vislumbran entre ellas.

En primer lugar se distinguen tres categorías que representan factores inherentes a la práctica de la danza, ya que están presentes durante su ejecución. Estas categorías son:

- Atención plena: implica enfocar la atención en el movimiento y en los detalles y matices de lo percibido a través de los sentidos como consecuencia de dicho movimiento. Estar alerta a lo que pasa dentro de sí durante el movimiento y estar receptivo a lo que pueda estar ocurriendo fuera y a las sensaciones que eso produce.

- Insight corporal: entendimiento al que tiene acceso la persona mediante la concientización de las sensaciones y los cambios en su cuerpo debido al movimiento. Refiere a la capacidad de comprender aspectos de sí mismo en el movimiento y de notar los pequeños cambios que este genera. No necesariamente está asociado a un proceso reflexivo sino que puede ocurrir por un ligero cambio en la percepción del movimiento, implicando a su vez una modificación en la autopercepción y la percepción del entorno.

- Libertad de movimiento: refiere a la posibilidad que tiene la persona para moverse libremente, sin necesidad de realizar pasos específicos o previamente determinados. La libertad de movimiento parece lograrse debido a: 1) la ausencia del concepto de error, lo que propicia que la persona se permita experimentar sin considerar que existen determinadas formas de hacer en el movimiento que serán catalogadas como correctas o incorrectas, dando paso a la expresión propia y genuina y a la apertura hacia la capacidad creadora y 2) la posibilidad de improvisación, que brinda espacio para el reconocimiento de la propia manera de moverse y de la propia manera de expresión, así como para la exploración y búsqueda de nuevos caminos para hacer y la posibilidad de modificarlas o adaptarlas.

Se han identificado también categorías que revelan procesos que ocurren en las personas que practican danza, los cuales se activan debido a la influencia de los factores antes descritos. Entre estas categorías se encuentran:

- Actualización del potencial: la danza es un medio para experimentar, al propiciar la ampliación de la percepción de nuevas sensaciones y vivencias. Brinda una oportunidad para adquirir un conocimiento experiencial de sí mismo y de sí mismo en relación con el mundo. Se vuelve una oportunidad para conocer.

- Despertar de la vitalidad: la danza propicia que las personas noten su fuerza vital mediante la experiencia que se obtiene a través del movimiento. La vitalidad que despierta la práctica de la danza está asociada a que la persona contacta con sus sentidos y la posibilidad de apreciar diversas sensaciones internas y externas, alcanzando una experiencia cada vez más amplia y rica en detalles y contactando con la sensación de estar vivo.

- Consciencia de sí mismo: la práctica de la danza conlleva una toma de contacto consigo mismo y el entorno que ocurre como consecuencia del movimiento. Implica ser consciente del propio cuerpo, de los propios movimientos y de las sensaciones. Es un 
volver la mirada hacia sí mismo y observar la propia manera de desenvolverse en el mundo.

- Conexión con otros: mediante la práctica de la danza se genera interconexión entre las personas, lo cual parece ser consecuencia de la conexión que la persona logra establecer consigo misma al concientizar las propias sensaciones y la propia experiencia y de la apertura y la disposición a mostrar aspectos de sí mismos y a compartir de manera genuina, conllevando a su vez un reconocimiento y respeto del otro y su vivencia.

- Facilitación de la expresión y comunicación: la consciencia de sí mismo que se logra en la práctica de la danza permite contactar con las propias emociones que, al estar encarnadas en el cuerpo, son susceptibles de ser evocadas mediante el movimiento. A su vez, el movimiento sirve como canal para la expresión de dichas emociones. Se convierte en un medio que le permite a la persona ampliar su lenguaje y la posibilidad de comunicación más allá de lo verbal, ganando recursos para la expresión.

Por último se presentan categorías que refieren a las consecuencias globales que experimentan las personas que practican danza, entre las que se han identificado las siguientes:

- Expansión del yo: las personas que practican danza experimentan un cambio en la percepción de sí mismos, asociado a un reconocimiento de las propias habilidades y la posibilidad de crear nuevas maneras de moverse y de existencia en el mundo. Este aspecto implica: 1) trascendencia de barreras reales (discapacidad física) o autoimpuestas (creencias), ya que la persona, al practicar danza, se abre a hacer cosas nuevas y probar nuevos movimientos, expandiendo los propios límites, transcendiéndolos en el hacer y experimentando una sensación de que puede llegar a lugares físicos que no se había planteado antes por la creencia de que no sería capaz, y 2) ampliación de la percepción de las propias capacidades, ocurriendo que la persona empieza a modificar la visión que tiene de sí mismo incluyendo nuevos aspectos en su autopercepción, bien porque va obteniendo avances en la ejecución de la actividad o porque se descubre realizando movimientos y experimentando sensaciones nuevas, y se genera un aumento de la valía personal.

- Sensación de integración: mediante el proceso de atención plena durante la práctica de danza se propicia la integración entre la mente y el cuerpo. Se lleva el pensamiento al cuerpo mediante la toma de consciencia de lo que está sucediendo en este. Así mismo, para poder realizar determinados movimientos o improvisaciones, existe una intención de hacer o moverse de determinada manera y, aunque no ocurra un análisis detallado para tomar decisiones sobre lo que se ejecutará, la mente está alerta para generar las respuestas necesarias. Esto brinda a la persona una sensación de unidad al poder integrar las sensaciones del cuerpo y la actividad mental mediante acciones expresivas. 


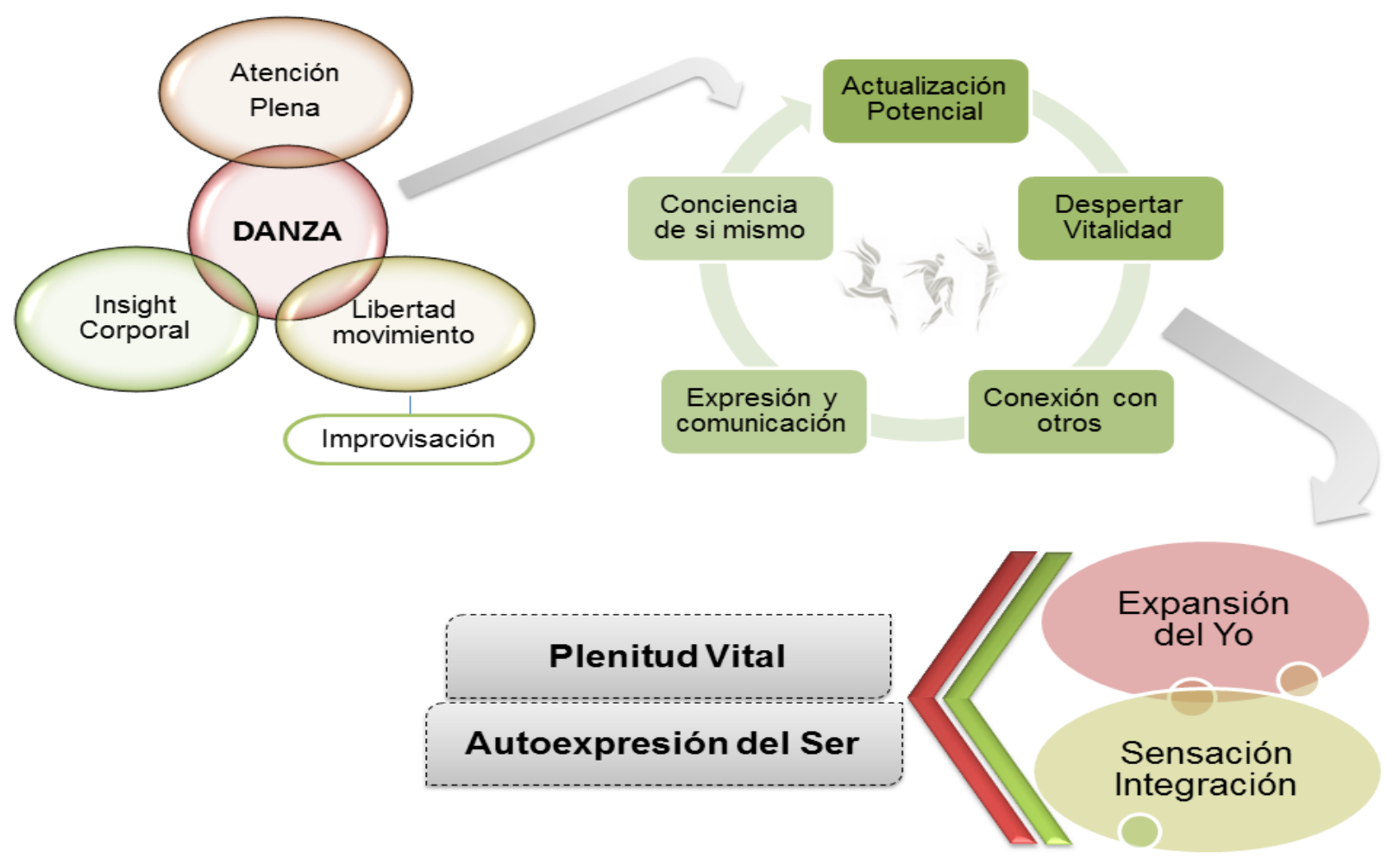

Figura 2. Relación entre categorías

Tabla 1. Categorías, indicadores y propiedades

\begin{tabular}{|c|c|c|}
\hline \multicolumn{2}{|r|}{ Categorías } & Propiedades \\
\hline \multirow{3}{*}{ 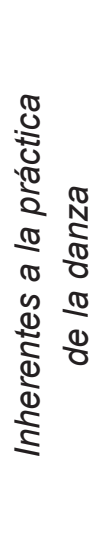 } & Atención plena & $\begin{array}{l}\text { - Atención focalizada en movimiento y sensaciones. } \\
\text { - Estado de alerta a sensaciones internas y externas. }\end{array}$ \\
\hline & Insight corporal & $\begin{array}{l}\text { - Entendimiento generado por concientización de sensaciones y cam- } \\
\text { bios en el propio cuerpo. } \\
\text { - Compresión de aspectos de sí mismo. } \\
\text { - Cambio en la percepción del propio movimiento. }\end{array}$ \\
\hline & $\begin{array}{l}\text { Libertad de movi- } \\
\text { miento }\end{array}$ & $\begin{array}{l}\text { - Moverse sin seguir pasos predeterminados. } \\
\text { - Ausencia de concepto de error. } \\
\text { - Posibilidad de improvisación: exploración y búsqueda de maneras } \\
\text { diferentes de acción y movimiento. }\end{array}$ \\
\hline
\end{tabular}




\begin{tabular}{|c|c|c|}
\hline \multirow{5}{*}{ 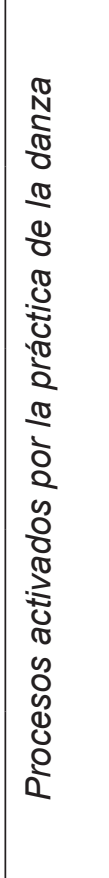 } & $\begin{array}{l}\text { Actualización del } \\
\text { potencial }\end{array}$ & $\begin{array}{l}\text { - Percepción de nuevas sensaciones y vivencias. } \\
\text { - Adquisición de conocimiento experiencial de sí mismo. } \\
\text { - Oportunidad para conocer. }\end{array}$ \\
\hline & $\begin{array}{l}\text { Despertar de vitali- } \\
\text { dad }\end{array}$ & $\begin{array}{l}\text { - Notar la propia fuerza vital en experimentación del movimiento. } \\
\text { - Ampliación de gama de detalles sentidos y percibidos. } \\
\text { - Sensación de estar vivo. }\end{array}$ \\
\hline & $\begin{array}{l}\text { Consciencia de sí } \\
\text { mismo }\end{array}$ & $\begin{array}{l}\text { - Consciencia del propio cuerpo, de movimientos y sensaciones pro- } \\
\text { pios. } \\
\text { - Dirigir la mirada hacia sí mismo. }\end{array}$ \\
\hline & Conexión con otros & $\begin{array}{l}\text { - Disposición a mostrar aspectos de sí mismos. } \\
\text { - Apertura a compartir con otros de manera genuina, respetando y reco- } \\
\text { nociendo su vivencia. }\end{array}$ \\
\hline & $\begin{array}{l}\text { Facilitación de } \\
\text { expresión y comu- } \\
\text { nicación }\end{array}$ & $\begin{array}{l}\text { - Evocación de emociones mediante movimiento. } \\
\text { - Movimiento como canal para expresar emociones. } \\
\text { - Ampliación del lenguaje y de recursos para expresión. }\end{array}$ \\
\hline \multirow{2}{*}{ 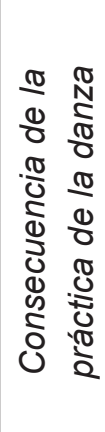 } & Expansión del yo & $\begin{array}{l}\text { - Cambio en la percepción de sí mismos. } \\
\text { - Reconocimiento de habilidades y capacidades creativas propias. } \\
\text { - Trascendencia de barreras reales (discapacidad física) o autoimpues- } \\
\text { tas (creencias limitantes). }\end{array}$ \\
\hline & $\begin{array}{l}\text { Sensaciones de } \\
\text { integración }\end{array}$ & $\begin{array}{l}\text { - Llevar el pensamiento al cuerpo mediante toma de consciencia de } \\
\text { movimiento y sensaciones. } \\
\text { - Mente alerta para generar respuesta de acción y movimientos. } \\
\text { - Integración mente-cuerpo en acciones expresivas. }\end{array}$ \\
\hline
\end{tabular}

\section{Discusión y conclusiones}

Los resultados obtenidos muestran un conjunto de categorías relacionadas con el bienestar psicoemocional y la plenitud vital, observándose que la práctica de la danza actúa como facilitador de la autoexpresión.

Las categorías encontradas se relacionan con los conceptos de experiencias cumbres (Maslow, 1972) y de estado de flow (Csikszentmihalyi, 1975). Específicamente, los factores de atención plena e insight corporal hacen referencia a un estado experimentado por aquellos que practican danza que se muestra congruente con la definición de estado de flow, en el que se enfoca la atención en una meta clara y definida, donde la persona se siente envuelta, concentrada, absorbida por la tarea y las horas pasan casi sin notarlo (Csikszentmihalyi, 1998).

Estas experiencias cumbres o de flow denotan estados intensos de júbilo o gozo, en los que la persona puede expresar de un modo unificado la vitalidad de ser, en un sentido genuino (Carrero, 2007), coincidiendo con lo encontrado en los resultados donde se puede ver que las personas experimentan una sensación de integración como consecuencia de la práctica de la danza.

Los factores inherentes a la danza y los procesos que estos activan promueven la experimentación de los estadios críticos en la manifestación de expresión del ser (Carrero, 2007). Las categorías referentes a «actualización del potencial» y «expansión del yo» se ven relacio- 
nadas con el primer estadio en donde la persona atraviesa un estado de yo resultado, experimentando una vivencia de extrañamiento. Ocurre una sensación nueva, de disfrute y realización extraordinaria que provoca un descolocamiento en la percepción que la persona tiene de sí misma, caracterizado por la sorpresa y la novedad. La emergencia de procesos de autoexpresión genera un estado de intenso placer, a la vez que se da cuenta de su nivel de competencia y maestría en la actividad que realiza, en este caso específico, la danza.

El factor de «libertad de movimiento» dentro de la danza facilita la entrada en el segundo estadio crítico en la expresión del ser, el estado de yo proceso. En este ocurre un estado de despertar, en donde el ser se percibe a través de un yo que descubre, para el que el proceso es más importante que los resultados. En este estado ocurre una completa absorción y el ser se exalta en su condición genuina sin necesidad de mediadores, desaparece la distancia entre el sujeto y la acción, no es necesario interpretar las realizaciones, la persona puede ser ella misma y la alegría expresada como goce intenso emerge, dando sentido, potencia y distintividad a las actividades que se emprenden (Carrero, 2007).

En este punto parece tener un papel importante la improvisación dentro de la danza, permitiendo que la persona pueda descubrir nuevas maneras de moverse, de accionar y de aproximarse a otros, sin apegarse a una determinada manera de danzar y dándose la oportunidad de experimentar con formas diferentes, lo que le permite validar su propia manera de ser a través del movimiento, sin juzgarla como correcta o incorrecta.

La toma de consciencia de sí mismo que se activa en la práctica de la danza facilita el autoconocimiento, aspecto esencial para alcanzar una vida plena. Desde la experiencia vivenciada de ser, la persona siente la conexión vital y genuina de un ser humano con sentido (Carrero, 2007). Dichos aspectos se revelan en las categorías emergidas sobre «despertar de la vitalidad» y "sensación de integración» que experimentan aquellos que practican danza. Estas categorías, vinculadas con las demás señaladas en los resultados, facilitan la sensación de bienestar al permitir que se produzcan estados de flow o cumbres, que contribuyen a la expresión genuina del yo. Todo ello permite el desarrollo de procesos de autoexpresión, en la actualización del potencial del ser.

La relación que se observa entre la danza y los conceptos de estados de flow y autoexpresion del ser indica su idoneidad como herramienta en la prevención de riesgos psicosociales y la promoción del bienestar. A través de la danza se pueden potenciar recursos personales que permitan hacer frente a la erosión de los dinamismos vitales, la fragilidad cultural para dar sentido a la vida, el desánimo y el debilitamiento de la confianza, aspectos que conllevan situaciones personales de marginación (García Roca, 1993, c. p. Agost, García y Calvo, 2010).

Las categorías expuestas como resultados presentan una saturación teoría parcial, siendo una de las limitaciones del presente estudio, por lo que las conclusiones obtenidas solo muestran las tendencias hasta ahora observadas a raíz del análisis de los datos. Se requiere seguir indagando para lograr la saturación total de las categorías y sus propiedades, dar oportunidad al surgimiento de nuevas categorías y determinar de manera más clara la interrelación existente entre ellas. Investigaciones futuras deberían indagar en el papel de la improvisación dentro de la danza como medio para promover la libertad de movimiento y la vivencia de experiencias cumbres o de flow, así como su influencia en la autoexpresión del ser y la actualización del potencial. 


\section{Referencias bibliográficas}

Abad, M., Castillo, E. y Orizia, A. (2014). Los efectos de un programa motor basado en la biodanza en relación con parámetros de inteligencia emocional en mujeres. Cuadernos de Psicología del Deporte, 14, 13-22.

Agost, R., García, R. y Calvo, H. (2010). Intervención comunitaria. Familia en situación de riesgo, discapacidad, adicciones y necesidades educativas especiales. En Francisco Juan García Bacete, Antonio V. Vaquer Chiva y Cristina Gommis Bru (coords.), Intervencion y mediación familiar: manual docente del máster de la Universitat Jaume I. Castellon de la Plana: Publicaciones de la Universitat Jaume I.

Carrero Planes, V. (2007). El sentido de "ser." Revista Electronica de Motivación y Emoción, 10, 26-27.

Carrero, V., Soriano, R. y Trinidad, A. (2012). Elementos de la Teoría fundamentada. Teoría fundamentada. 2. ${ }^{a}$ ed. Madrid: Centro de Investigaciones Sociológicas.

Csikszentmihaly, M. y Csikszentmihaly, I. (1998). Experiencia óptima. Estudios psicológicos del flujo en la conciencia. Bilbao: Desclee De Brouwer.

Csikszentmihalyi, M. (1975). Beyond boredom and anxiety: The experience of flow in work and play. San Francisco: Jossey-Bass.

Glaser, B. S. y Strauss, A. (1967). The discovery of grounded theory. Chicago: Aldine. 